\title{
CYP1A2 - a novel genetic marker for early aromatase inhibitor response in the treatment of breast cancer patients
}

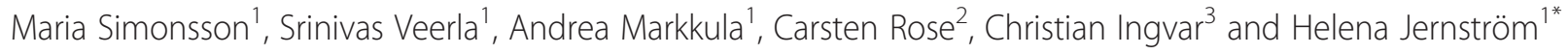

\begin{abstract}
Background: Endocrine resistance is a major obstacle to optimal treatment effect in breast cancer. Some genetic markers have been proposed to predict response to aromatase inhibitors (Als) but the data is insufficient. The aim of the study was to find new genetic treatment predictive markers of Als.

Methods: The ongoing population-based BC-blood study in Lund, Sweden includes women with primary breast cancer. This paper is based on Al-treated patients with estrogen receptor positive tumors who underwent breast cancer surgery in 2002-2008. First, an exploratory analysis of 1931 SNPs in 227 genes involved in absorption, distribution, metabolism, and elimination of multiple medications, using DMET ${ }^{\mathrm{TM}}$ chips, was conducted in a subset of the cohort with last follow-up in December $31^{\text {st }} 2011$ (13 cases, 11 controls). Second, selected SNPs from the first analysis were re-analyzed concerning risk for early breast cancer events in the extended cohort of 201 Al-treated with last follow-up in June $30^{\text {th }}$ 2014. Clinical data were obtained from medical records and population registries.
\end{abstract}

Results: Only CYP1A2 rs762551 C-allele was significantly associated with increased risk for early events in the 24 patients $(P=0.0007)$ and in the extended cohort, adjusted Hazard ratio (HR) 2.22 (95\% Cl 1.03-4.80). However, the main prognostic impact was found within five years, adjusted HR $7.88(95 \% \mathrm{Cl}$ 2.13-29.19). The impact of the CYP1A2 rs762551 C-allele was modified by a functional polymorphism in the regulator gene AhR Arg554Lys ( $\mathrm{G}>\mathrm{A}$ ). Compared to patients who were homozygous for the major allele in both genes (CYP1A2 A/A and AhR G/G), a 9-fold risk for early events was found in patients who had at least one minor allele in both genes, adjusted HR 8.95 (95\% Cl 2.55-31.35), whereas patients with at least one minor allele in either but not both genes had a 3-fold risk for early events, adjusted HR 2.81 (95\% Cl 1.07-7.33). The impact of CYP1A2 rs762551 C-allele was also modified by the CYP19A1 rs4646 C/C, adjusted HR 3.39 (95 \% Cl 1.60-7.16) for this combination. This association was strongest within the first five years, adjusted HR 10.42 (95 \% Cl 3.45-31.51).

Conclusion: CYP1A2 rs762551 was identified as a new potential predictive marker for early breast cancer events in Al-treated breast cancer patients. Moreover, combined genotypes of CYP1A2 rs762551 and CYP19A1 rs4646 or AhR Arg554Lys could further improve prediction of early Al-treatment response. If confirmed, these results may provide a way to more personalized medicine.

Keywords: Breast cancer, CYP1A2, CYP19A1, AhR, Polymorphisms, Treatment response, Aromatase inhibitor

\footnotetext{
* Correspondence: helena.jernstrom@med.lu.se

'Department of Clinical Sciences, Lund, Division of Oncology and Pathology,

Lund University, Lund, Sweden

Full list of author information is available at the end of the article
} 


\section{Background}

Breast cancer is one of the leading causes of cancer morbidity and mortality among women worldwide [1]. The majority of breast cancer patients have tumors that express hormone receptors [2,3] and can thus be offered endocrine therapy such as tamoxifen and aromatase inhibitors (AIs). However, endocrine resistance is a major obstacle to optimal treatment effect [4]. Several genetic markers for tamoxifen response have been proposed, although no consensus has yet been reached [5-11]. For AIs, data on genetic markers are sparse [10-13]. The response rates to AIs vary between 35 and $70 \%$ in the neoadjuvant setting $[4,14,15]$ and may be lower in advanced disease [16]. By identifying mechanisms of resistance as well as treatment predictive factors, patients may be offered more effective personalized medicine and be spared side-effects of ineffective treatment [17].

Only a few studies have investigated the association between polymorphisms in Cytochrome P450 (CYP) CYP19A1 (aromatase) and disease-free survival in breast cancer $[10,18,19]$. There are currently only a few studies published with a proposed polymorphism for predicting AI response in the adjuvant setting, and these have contradictory results $[11,13]$. Some studies have investigated the impact of CYP19A1 polymorphisms on treatment response in the metastatic- [20] and in the neoadjuvant settings $[21,22]$. However, the results have been inconsistent. Therefore, it is currently unknown whether single nucleotide polymorphisms (SNPs) in CYP19A1 are associated with a risk of early events in patients treated with $\mathrm{AI}$ as first line treatment.

The formation and metabolism of estrogens in the steroidal sex hormone metabolism is complex and involves several enzymes. In addition to CYP19A1, some examples include CYP1A1, CYP1A2, COMT, and CYP3A4 [23]. Several of these enzymes are also involved in the metabolism of AIs [24, 25]. Furthermore, AIs interfere with some of these enzymes; letrozole has been shown to inhibit CYP2A6 and CYP2C19 in vitro [26], anastrozole has been shown to inhibit CYP1A2, CYP2C9, and CYP3A in vitro [27], and exemestane has been shown to be metabolized by CYP4A11 and CYP1A1/2 in vitro [28]. Polymorphisms in the corresponding genes may be a mechanism behind primary (de novo) resistance of $\mathrm{AI}$ as estrogens are known risk factors for recurrence of breast cancer and the enzymes that metabolize estrogens are tightly linked to AI metabolism. Two of these genes involved in estrogen metabolism, CYP1A1 and CYP1A2, share a common promoter [29] and are under regulatory control of the aryl hydrocarbon receptor (AhR) [30]. These genes may therefore be of interest to study in relation to AI response.

To find new markers beyond the candidate genes for AI resistance, it might be useful to expand the search to other known genes involved in Absorption, Distribution, Metabolism, and Elimination (ADME-related genes). High-throughput, drug metabolism enzymes and transporters $\left(\mathrm{DMET}^{\mathrm{TM}}\right)$ chips genotype several SNPs at the same time [31]. The Affymetrix DMET Plus Premier Pack includes 1931 SNPs in 227 genes in ADME-related genes on a single array. We hypothesized that SNPs in the aromatase gene CYP19A1 and SNPs in other genes for drug and estrogen metabolism may be used as treatment predictive markers for adjuvant treatment with AI in primary breast cancer patients. The aim of the study was: 1) to perform an exploratory analysis using the $\mathrm{DMET}^{\mathrm{\tau} \mathrm{M}}$ chip to find new treatment predictive markers in a subset of the cohort and 2) to examine these potential markers with a special focus on CYP19A1 in relation to a risk for early events in the extended cohort of AI-treated breast cancer patients.

\section{Methods \\ Study population}

Women diagnosed with a primary breast cancer at the Skåne University Hospital in Lund, Sweden were invited preoperatively to participate in an ongoing prospective population-based cohort-the BC-blood study. Patients with a prior history of another cancer diagnosis within the last ten years were not enrolled. The overall aims of the BC-blood study are to elucidate factors that may have prognostic or predictive value. This paper is based on data collected from 634 primary breast cancer patients between October 2002 and October 2008. Patients were followed from inclusion to the first breast cancer event or distant metastasis, respectively, and patients without events were censored at the last follow-up or death prior to July $1^{\text {st }} 2014$. As previously described, the follow-up rates of the patients were high [32]. During the time the cohort was compiled, 1090 patients went through breast cancer surgery and approximately $58 \%$ of these patients were included [33]. A lack of research nurses explains most of the patients who were missed and approximately $5 \%$ of the patients were missed due to unverified diagnosis at the time of surgery. Written informed consent was obtained from all patients, and the study was approved by the ethics committee of Lund University (Dnr LU75-02, LU37-08, and LU658-09).

Breast cancer events included ipsilateral, contralateral, axillary lymph node, and distant metastases. Information concerning breast cancer events was obtained from patient charts, pathology reports, and the Regional Tumor Registry. The date of death was obtained from the Swedish Population Registry. The first breast cancer event of any type was considered the primary endpoint, and distant metastasis was considered a secondary endpoint. Breast cancer treatment was prescribed according to the standard of care at 
Skåne University hospital. Information regarding the type of adjuvant treatment was collected from patient charts and questionnaires. Treatment data were registered up to the last follow-up prior to any event. Data on tumor size, histological type and grade, and number of involved axillary lymph nodes were obtained from each patient's pathology report. The tumors were analyzed at the Department of Pathology at Lund University Hospital. Estrogen receptor (ER) and progesterone receptor (PgR) status were determined as previously described $[5,34]$. The patients completed questionnaires preoperatively and at multiple times postoperatively. The questionnaires included questions such as reproductive history, use of exogenous hormones, smoking history, and any medications used during the past week as previously described [35]. During the preoperative visit, a research nurse collected blood samples for genotyping. The blood was collected and centrifuged and the samples were frozen at $-80^{\circ} \mathrm{C}$ within two hours.

\section{Genotyping}

Genomic deoxyribonucleic acid (DNA) was extracted from the patients' leukocyte portion of frozen peripheral blood using the Wizard Genomic DNA Purification Kit (Promega, Madison, USA) or Quickgene-610 L and Quickgene-810 (Fujifilm life science, Science imaging $\mathrm{AB}$, Scandinavia). The samples were then genotyped using the DMET ${ }^{\mathrm{m}}$ (Drug Metabolizing Enzymes and Transporters) Plus Premier Pack, which is a microarray assay developed by Affymetrix (Santa Clara, CA, USA), according to the manufacturer's instructions. The DMET ${ }^{\text {тм }}$ experimental analysis was performed at SCIBLU Genomics at Lund University.

Genotyping was also performed at the Region Skåne Competence Centre (RSKC Malmö), Skåne University Hospital, Malmö, Sweden. The CYP19A1 SNPs rs700518, rs4646, Aro1 (rs4775936), Aro2 (rs10459592), and two functional AhR SNPs Arg554Lys (rs2066853) and Val570Ile (rs4986826) were analyzed with matrix-assisted laser desorption/ionization time-of-flight mass spectrometry on a Sequenom MassARRAY ${ }^{\oplus}$ platform (Sequenom, San Diego, CA, USA), using iPLEX reagents according to the manufacturers' protocol. The Sequenom MassARRAY $^{\circledast}$ software was used for multiplex SNP analysis design. The rs700518 SNP was not successfully genotyped. The rs10046 SNP and the CYP1A2*1F (rs762551) SNP were genotyped using a Taqman SNP allelic discrimination assay in 384-well format on an ABI PRISM 7900 Sequence Detection System (Applied Biosystems, Foster City, CA, USA). Over $10 \%$ of the samples were run in duplicates with a concordance of $100 \%$.

There were 19 patients not successfully genotyped for the CYP1A2*1 F (rs762551) SNP using TaqMan. For 16 of these patients, rs762551 genotypes were available from DNA sequencing from a previous study [18]. The concordance rate between the two methods was $99.8 \%$. Haplotypes of CYP19A1 were constructed by crosstabulation of the genotypes of the CYP19A1 SNPs. This resulted in nine haplotypes. Linkage disequilibrium (LD) was observed between rs4646 and rs10046 ( $r=0.68)$, and between rs10046 and Aro1 $(r=0.90)$, as well as between Aro1 and Aro2 $(r=0.79)$. Therefore, the 14 missing genotypes for rs10046 could be imputed. The minor allele was defined according to the Database of Single Nucleotide Polymorphisms (dbSNP) [36].

\section{Data analyses}

The analyses of data from the DMET ${ }^{\mathrm{TM}}$ chip were performed using the $\mathrm{DMET}^{\mathrm{TM}}$ console software. The samples with QC call rates $\geq 99 \%$ were considered for further analyses. The analysis included 13 patients with breast cancer events who had been treated with AI but not with chemotherapy prior to the event by December $31^{\text {st }}$ $2011(n=13)$. The controls were 11 AI-treated patients without chemotherapy or tamoxifen and without recurrence who had a follow-up time of at least five years with last follow-up December $31^{\text {st }} 2011$. Fisher's exact test was used, and to make allowances for multiple testing, a $P$-value $<0.005$ was considered significant. This $P$-value allows for $0.5 \%$ of the findings to be false positive.

Statistical survival analyses of the extended cohort were performed with IBM SPSS Statistics, version 19.0 (IBM Corp. Armonk, NY, USA). A flowchart of patients included in the final survival analyses is presented in Fig. 1. After exclusion, 201 AI-treated patients were included in the analyses. In total, 32 patients were diagnosed with some type of breast cancer event during the 11-year follow-up period. Of these, 22 presented with distant metastases. A Kaplan-Meier LogRank test was used for univariable analyses of the risk of early events in relation to the different genotypes, haplotypes, and diplotypes of the SNPs. Since few patients had an invasive tumor size $\geq 51 \mathrm{~mm}$ or muscular or skin involvement, these patients were combined with the patients with invasive tumor sizes between 21 and $50 \mathrm{~mm}$ in the multivariable analyses. Regular smokers and occasional smokers were classified as current smokers. Cox regression was used to calculate Hazard Ratios (HRs) in relation to the SNPs after adjusting for age (linear), invasive tumor size $(<21 \mathrm{~mm}$ versus $\geq 21 \mathrm{~mm}$ or skin or muscular involvement independent of size), any axillary lymph node involvement (yes/no), histological grade III (yes/no), preoperative smoking status (yes/no), body mass index (BMI) $\geq 25 \mathrm{~kg} / \mathrm{m}^{2}$ (yes/no) radiation therapy (yes/no), chemotherapy (yes/no), and tamoxifen therapy (yes/no). A $P$-value $<0.05$ was considered significant. All $P$-values were two-tailed. Nominal $P$-values are presented without 


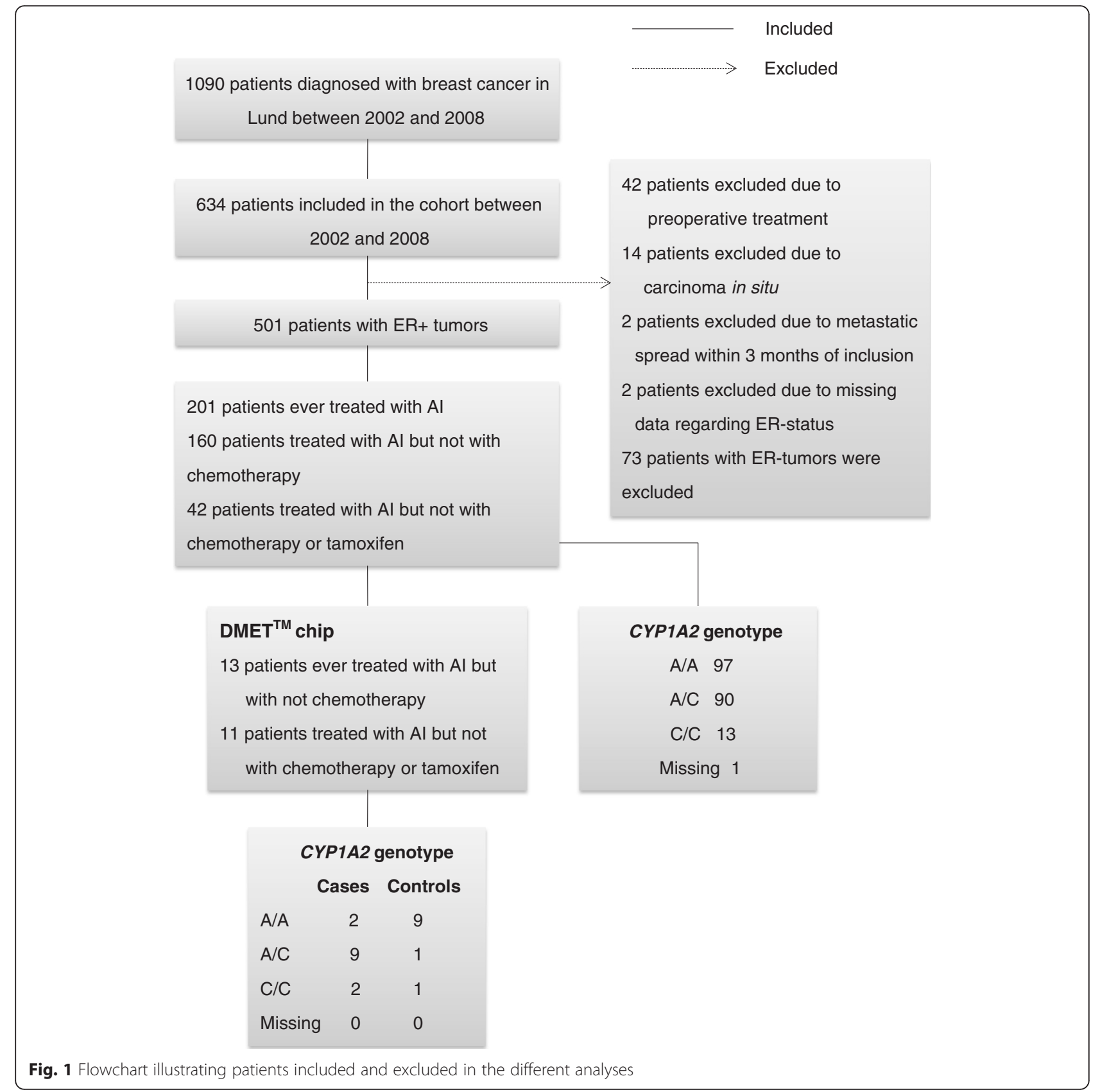

adjustments for multiple testing since this is an exploratory study [37]. A prior power calculation assuming a study with 200 patients; $50 \%$ of the patients had a major allele and an accrual interval of 6 years with additional follow-up after the accrual interval of 4 years, showed that the study could detect true HRs of failure for patients homozygous for the major allele relative to patients with variant alleles of 0.603 or 1.824 with $80 \%$ power and a type 1 error probability of 0.05 [38]. The study is based on the REMARK (Reporting Recommendations for Tumor Marker Prognostic Studies) criteria [39].

\section{Results}

Patient characteristics, tumor characteristics, and Al treatment

Patient and tumor characteristics of the patients are presented in Tables 1 and 2, respectively. There were no substantial differences in the characteristics between the AI-treated patients in the extended cohort and the patients analyzed with the DMET ${ }^{\mathrm{sin}}$ chip other than age and height. The distribution of AIs was as follows: anastrozole $67.2 \%$, letrozole $26.4 \%$, exemestane $5.0 \%$, anastrozole and letrozole in sequence $1.0 \%$, and AI type missing $0.5 \%$. 
Table 1 Patient characteristics of the Al-treated patients with ER+ tumors included in the DMETM chip analysis and the extended cohort

\begin{tabular}{|c|c|c|c|c|}
\hline & \multicolumn{2}{|c|}{$\begin{array}{l}\text { Patients included in the analysis of the } \\
\qquad \begin{array}{c}\text { DMET } \\
n=24\end{array}\end{array}$} & \multicolumn{2}{|c|}{$\begin{array}{l}\text { Patients in the extended cohort included in the } \\
\qquad \begin{array}{l}\text { survival analyses } \\
n=201\end{array}\end{array}$} \\
\hline & Median (IQR) or \% & Missing & Median (IQR) or \% & Missing \\
\hline Age at diagnosis, yrs & $67.7(60.0-72.7)$ & 0 & $60.9(54.4-66.4)$ & 0 \\
\hline Weight, kgs & $70.0(61.7-82.10)$ & 0 & $70.0(64.0-79.0)$ & 2 \\
\hline Height, m & $1.64(1.58-1.68)$ & 0 & $1.66(1.62-1.70)$ & 0 \\
\hline $\mathrm{BMl}, \mathrm{kgs} / \mathrm{m}^{2}$ & $26.6(23.6-30.6)$ & 0 & $25.2(23.2-28.8)$ & 2 \\
\hline Age at menarche, yrs & $14.0(13.0-14.0)$ & 0 & $13.0(12.0-14.0)$ & 0 \\
\hline Parous, \% & $87.5 \%$ & 0 & $85.1 \%$ & 0 \\
\hline Age at first full-term pregnancy, yrs ${ }^{a}$ & $23.5(21.0-26.8)$ & 3 & $24.0(22.0-27.0)$ & 31 \\
\hline Ever use of oral contraceptives, \% & $62.5 \%$ & 0 & $70.6 \%$ & 0 \\
\hline Ever use of hormone therapy, \% & $45.8 \%$ & 0 & $54.0 \%$ & 1 \\
\hline Current smoker prior to surgery, $\%$ & $20.8 \%$ & 0 & $17.4 \%$ & 0 \\
\hline Alcohol abstainers & $16.9 \%$ & 0 & $10.0 \%$ & 1 \\
\hline Preoperative daily coffee consumption $2+$ cups/day & $75.0 \%$ & 0 & $83.5 \%$ & 1 \\
\hline
\end{tabular}

${ }^{\mathrm{a}}$ Of the parous patients

$\mathrm{DMET}^{\mathrm{TM}}$ analysis and selection of SNPs for further analyses Of the 1931 SNPs, 1911 were successfully genotyped. Only the CYP1A2*1F rs762551 C-allele was significantly associated with increased risk for early events among the 24 AI-treated patients $(P=0.0007)$. The CYP1A2 rs762551 was thus elected for analyses in the extended cohort. The CYP19A1 SNPs were not significantly associated with survival in the analyses of the

Table 2 Tumor characteristics of the Al-treated patients with ER+ tumors included in the DMET ${ }^{\mathrm{TM}}$ chip analysis and the extended cohort

\begin{tabular}{|c|c|c|c|c|}
\hline & \multicolumn{2}{|c|}{$\begin{array}{l}\text { Patients included in the analysis of the } \\
\qquad \begin{array}{c}\text { DMETM } \\
\text { chip }\end{array} \\
n=24\end{array}$} & \multicolumn{2}{|c|}{$\begin{array}{l}\text { Patients in the extended cohort included in the } \\
\text { survival analyses } \\
\qquad n=201\end{array}$} \\
\hline & Number (\%) & Missing & Number (\%) & Missing \\
\hline Invasive tumor size, mm (stage) & & 0 & & 0 \\
\hline$\leq 20(\mathrm{pT} 1)$ & $15(62.5 \%)$ & & $128(63.7 \%)$ & \\
\hline $21-50(\mathrm{pT})$ & $8(33.3 \%)$ & & $69(34.3 \%)$ & \\
\hline $51-(\mathrm{pT} 3)$ & $1(4.2 \%)$ & & $4(2.0 \%)$ & \\
\hline Skin or muscular involvement (pT4) & $0(0 \%)$ & & $0(0 \%)$ & \\
\hline$\geq 21 \mathrm{~mm}$ or skin or muscular involvement & $9(37.5 \%)$ & & $73(36.3 \%)$ & \\
\hline Axillary node involvement & & 0 & & 0 \\
\hline 0 & $8(33.3 \%)$ & & $55(27.4 \%)$ & \\
\hline $1-3$ & $9(37.5 \%)$ & & $107(53.2 \%)$ & \\
\hline $4+$ & $7(29.2 \%)$ & & 39 (19.4\%) & \\
\hline Any axillary lymph node & $16(66.7 \%)$ & & $146(72.6 \%)$ & \\
\hline Histological grade & & 0 & & 0 \\
\hline । & $6(25.0 \%)$ & & $44(21.9 \%)$ & \\
\hline$\|$ & $16(66.7 \%)$ & & $124(61.7 \%)$ & \\
\hline III & $2(8.3 \%)$ & & $33(16.4 \%)$ & \\
\hline Hormone receptor status & & 0 & & 0 \\
\hline ER+ & $24(100.0 \%)$ & & 201 (100.0\%) & \\
\hline $\mathrm{PgR}+$ & 20 (83.3 \%) & & $161(80.1 \%)$ & \\
\hline
\end{tabular}


DMET $^{\text {тM }}$ chip in the 24 patients. The first CYP19A1 SNP, rs700518, appeared in $12^{\text {th }}$ place $(P=0.014)$. However, a special focus was placed on CYP19A1 in this paper since aromatase is the target of AIs. Therefore, five CYP19A1 SNPs (rs700518, rs4646, rs10046, Aro1, and Aro2) were also selected for survival analyses in the extended cohort. Moreover, since $A h R$ is involved in the regulation of $C Y P 1 A 2$, genotyping was also performed for two functional AhR SNPs Arg554Lys (rs2066853) and Val570Ile (rs4986826) of which only Arg554Lys was included in the DMET ${ }^{\mathrm{mm}}$ chip.

\section{CYP1A2 rs762551 in relation to risk for early events in Al-treated patients}

The patients were followed for up to 11 years with a median follow-up time of 7.2 years (IQR 5.3-9.2) for patients who were alive and still at risk at the last followup. The minor allele frequency (MAF) was $29.0 \%$ for CYP1A2 rs762551 (C-allele). AI-treated patients with ER + tumors and any C-allele of CYP1A2 rs762551 genotype $(n=103)$ had a significantly higher risk for early breast cancer events versus the patients with $\mathrm{A} / \mathrm{A}$ genotype (Fig. 2a; adjusted HR 2.22 (95 \% CI 1.03-4.80). However, the main treatment predictive impact of CYP1A2 rs762551 was found within five years of inclusion (early events), adjusted HR 7.88 (95 \% CI 2.13-29.19).

When the patients ever treated with chemotherapy were excluded as was done in the DMET ${ }^{\mathrm{ma}}$ analysis, 159 patients remained and 26 events occurred until $30^{\text {th }}$ June 2014. Here, the association did not remain significant, adjusted HR 1.97 (95 \% CI 0.84-4.59) but a significant impact was found within five years of inclusion, adjusted HR 7.22 (95 \% CI 1.49-40.00).

After exclusion of patients ever treated with tamoxifen and/or chemotherapy, only 42 patients remained and 8 events occurred. The association was significant in the univariable model (LogRank $P=0.002$ ) and for events within five years $(P=0.032)$. Due to small numbers, no Cox regression was performed. The AI-treated patients with ER+ tumors and any C-allele of CYP1A2 rs762551 also had a significantly increased risk for early distant metastases overall (LogRank $P=0.020$ ), adjusted HR 3.47 (95 \% CI 1.26-9.56) and within five years (LogRank $P=0.020$ ), adjusted HR 7.80 (95 \% CI 1.51-40.32).

\section{Combination of CYP1A2 and AhR}

Genotyping of the AhR SNP Val570Ile (rs4986826) was non-informatory since all patients had the G/G genotype. The minor allele frequency for Arg554Lys (rs2066853 A-allele, Lys) was $11.9 \%$. There was no linkage between the CYP1A2 rs762551 and AhR Arg554Lys genotypes. AhR Arg554Lys was not associated with early events in the patients included in the analysis of the $\mathrm{DMET}^{\mathrm{TM}}$ chip and appeared in $524^{\text {th }}$ place. However, in the extended cohort, patients with any A-allele of the $A h R$ Arg554Lys had a significantly higher risk for early events compared to patients with the G/G genotype overall (LogRank $P=0.005)$, adjusted HR $2.61(95 \%$ CI 1.24-5.50) and within five years (LogRank $P=0.013$ ), adjusted HR 3.33 (95 \% CI 1.24-8.96).

There was no interaction between the CYP1A2 rs762551 and $A h R$ Arg554Lys. However, a combination of the two SNPs showed multiplicative associations. Patients who had at least one minor allele in both genes, i.e., any CYP1A2 C-allele and any $A h R$ A-allele, had the highest risk for early events followed by patients who had a $C Y P 1 A 2 \mathrm{~A} / \mathrm{A}$ genotype and $A h R$ any A-allele or CYP1A2 any $\mathrm{C}$-allele and $A h R$ G/G compared to patients who were homozygous for the major allele in both genes CYP1A2 A/A and AhR G/G (LogRank 3 df; $P=0.013)$, Fig. 3a. Since the curves for patients with $C Y P 1 A 2 \mathrm{~A} / \mathrm{A}$ and $A h R$ any A-allele or CYP1A2 any C-allele and $A h R$ G/G overlapped, these genotypes were combined in the multivariable model into one group of patients that were homozygous for the major allele in one but not both genes. Overall, compared to patients who were homozygous for the major allele in both genes, patients who had at least one minor allele in both genes ( $n=20,7$ events) had a 9-fold risk for early events, adjusted HR 8.95 (95 \% CI 2.55-31.35), whereas patients with at least one minor allele in one but not both genes ( $n=107,19$ events) had a 3-fold risk for early events, adjusted HR 2.81 (95 \% CI 1.07-7.33). These results were also seen when the analysis was restricted to the first five years (LogRank $3 \mathrm{df} ; P<0.001$ ), Fig. $3 \mathrm{~b}$.

\section{CYP19A1 in relation to risk for early events in Al-treated patients}

The CYP19A1 SNP rs700518 was not successfully genotyped using iPlex and could not be further analyzed. The MAF for rs4646 (A-allele), rs10046 (T-allele), Aro1 (Tallele), and Aro2 (T-allele) were 30.8, 49.3, 47.0 and $40.0 \%$, respectively. In line with the DMET ${ }^{\mathrm{mi}}$ data, the genotypes, haplotypes, and diplotypes of the four CYP19A1 SNPs were not associated with early events (all adjusted $P$-values $>0.10$; see Fig. $2 b$ for rs4646). Excluding the patients ever treated with chemotherapy and/or tamoxifen did not materially change the result. All of the results remained insignificant in relation to risk for distant metastases.

\section{Combination of CYP19A1 SNPs and CYP1A2}

To investigate whether the findings regarding risk for early events and the CYP1A2 rs762551 SNP were modified by the CYP19A1 SNPs, stratification according to each genotype of the four CYP19A1 SNPs was performed. No effect modification was observed between the strata for rs10046, Aro1, Aro2, and CYP1A2 


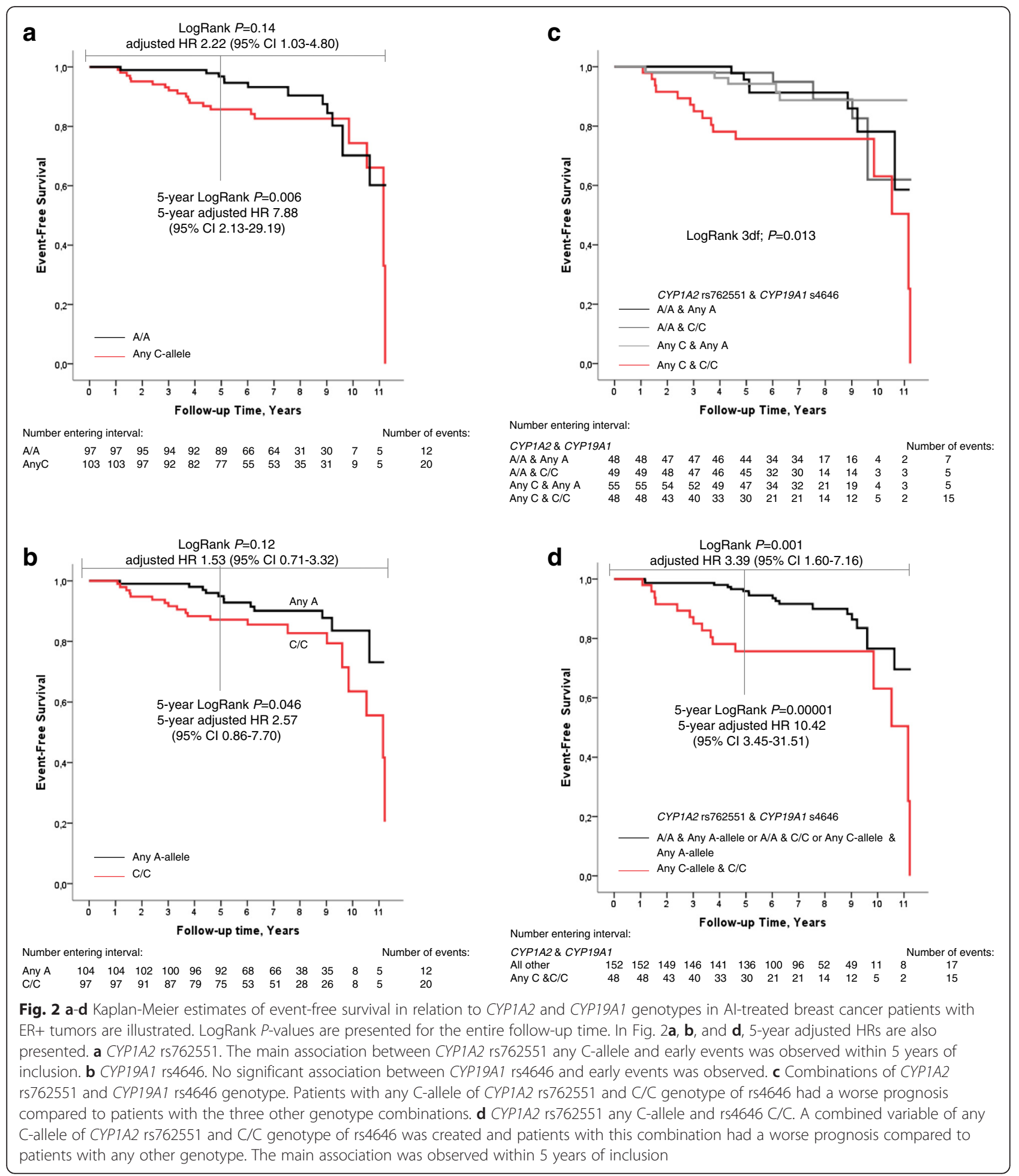

rs762551. The interaction analyses were non-significant. However, the interaction between CYP1A2 rs762551 any $\mathrm{C}$-allele and the $\mathrm{C} / \mathrm{C}$ genotype of CYP19A1 rs4646 was significant (adjusted $P_{\text {interaction }}=0.022$ ). Any C-allele carriers of CYP1A2 rs762551 with the $\mathrm{C} / \mathrm{C}$ genotype of CYP19A1 rs4646 ( $n=48,15$ events) had over a 3-fold increased risk of early events versus the rest of the AItreated patients (Fig. 2c-d; LogRank $P=0.001$ ), adjusted HR 3.39 (95 \% CI 1.60-7.16). As with CYP1A2 rs762551 alone, the main treatment predictive impact of was found within five years (LogRank $\mathrm{P}=0.00001)$, adjusted $\mathrm{HR}$ 10.42 (95 \% CI 3.45-31.51). 


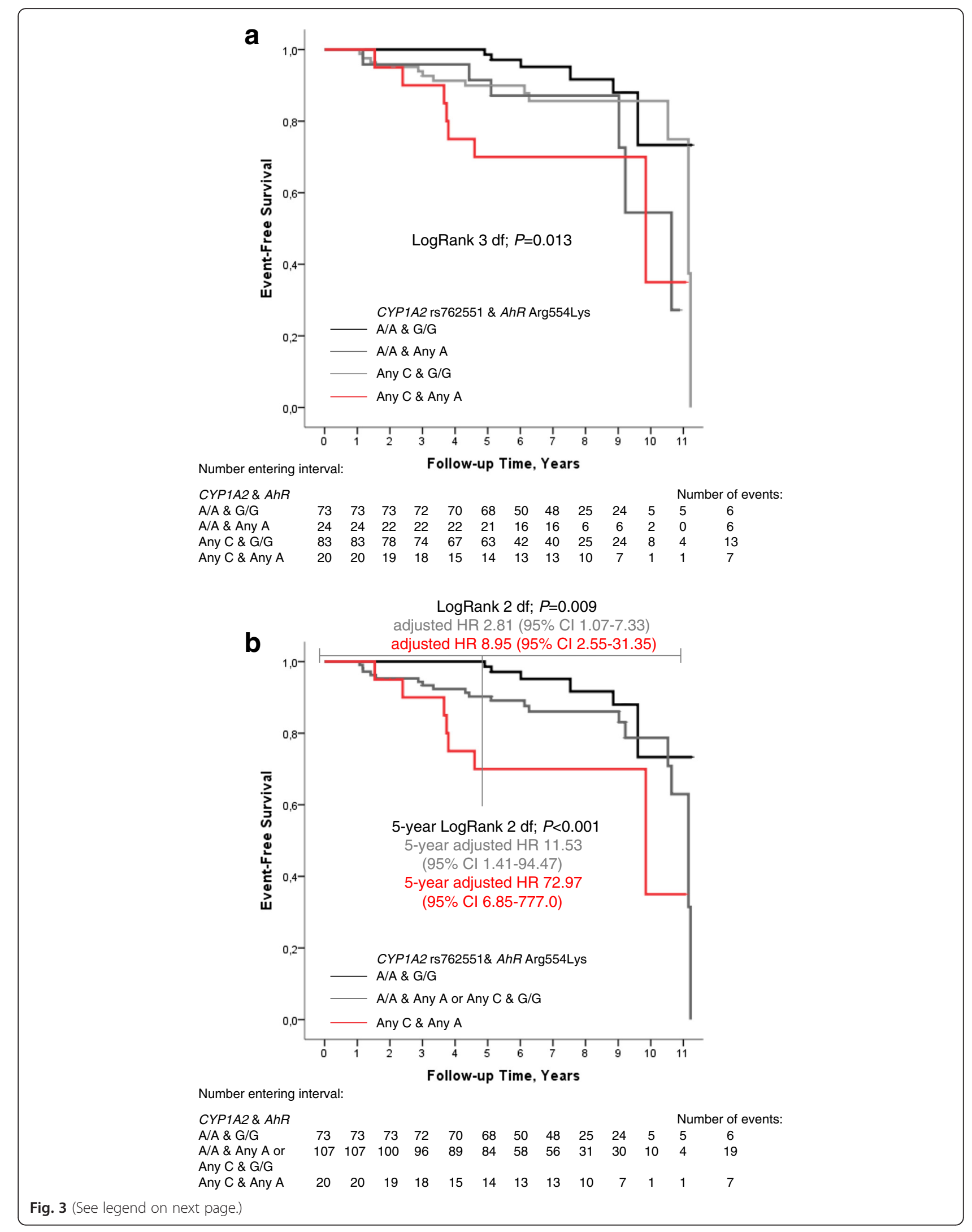


(See figure on previous page.)

Fig. 3 a Combinations of CYP1A2 rs762551 and Ahr Arg554Lys genotype. Patients with any C-allele of CYP1A2 rs762551 and any A-allele of AhR Arg554Lys had a worse prognosis followed by patients with at least one minor allele in either but not both genes and the lowest risk was seen in patients with the CYP1A2 rs762551 A/A genotype combined with the AhR Arg554Lys G/G genotype. b Three combinations of CYP1A2 rs762551 and Ahr Arg554Lys genotype. Patients who had a CYP1A2 A/A genotype and AhR any A-allele or CYP1A2 any C-allele and AhR G/G genotype were combined to one moderate risk group as the curves were similar in these groups. Patients with CYP1A2 rs762551 any C-allele and AhR Arg554Lys any A-allele had the highest risk for early events, followed by the combined group, compared to patients with CYP1A2 rs762551 A/A genotype and AhR Arg554Lys G/G genotype. Please note that there are fewer patients with longer follow-up times as this is an on-going cohort

\section{Discussion}

The present study investigated the association between SNPs in ADME-related genes and the risk of early breast cancer events in AI-treated patients with primary breast cancer. The main finding was that CYP1A2 rs762551 was significantly associated with risk of early breast cancer events among AI-treated patients with ER+ tumors, both in the exploratory analysis and in the extended cohort. This suggests that CYP1A2 rs762551 may be a predictive marker for early AI-response. To the best of our knowledge, this has not been reported before.

The DMET $^{\text {тм }}$ chip was selected because the included SNPs are involved in genes of importance for drug metabolism and transportation. This approach increases the chance that a finding is of biological relevance for AI response. The cut-off for the $P$-value in the DMET ${ }^{\mathrm{TM}}$ analysis was chosen to allow for identification of potentially new candidate genes while keeping the number of false positive findings low. As this was an exploratory analysis of nearly 2000 SNPs, a Bonferroni correction would have been too stringent and the risk for false negative findings substantial. The CYP1A2 rs762551 was the only SNP that met the predetermined cut-off and the enzyme is involved in the metabolic pathways of AIs or is inhibited by AIs $[25,27,28]$, which increases the chance that the finding may be of biological relevance.

CYP1A2 is a phase I pathway for drug metabolism and elimination [40]. An in vitro study reported a significant role of CYP1A2 in exemestane metabolism [28]. Moreover, CYP1A2 catalyzes the conversion of estradiol to hydroxylated metabolites, primarily 2-hydroxylated estradiol [23], which has been shown to act as a weak or even as an anti-estrogenic substance [41]. In a subset of 59 patients in the current cohort, the CYP1A2 rs762551 C-allele was associated with a low 2OHE-to16alphaOHE1 plasma ratio both pre- and postoperatively [42]. However, none of these patients were treated with AIs at the time of blood draw. Since AIs block estrogen formation, it is unlikely that there are measurable estrogen metabolite plasma levels in the 201 AI-treated patients.

The CYP1A2 rs762551 is located in intron 1 of the CYP1A2 gene and carries a $-163 C>A$ substitution. CYP1A1/2 expression is regulated by the AhR and a number of transcription factors and might be influenced by transcriptional coactivators and corepressors [43]. The A/A genotype of CYP1A2 rs762551 is highly inducible especially by smoking [44] and coffee consumption [45]. Neither smoking nor coffee consumption accounted for the association between CYP1A2, AhR, and risk for early events (data not shown). Furthermore, all multivariable models were adjusted for smoking. While the CYP1A2 rs762551 has been shown to influence inducibility, it has not been shown to significantly alter the gene expression [46]. The results are conflicting as to whether the SNP influences CYP1A2 enzyme activity $[43,46]$. Aklillu et al. have performed extensive characterization of CYP1A2 genotype phenotype correlations [47]. Cell transfection experiments showed that there was no significant difference in the constitutive transcriptional activity depending on the CYP1A2 rs762551 SNP. Further, electrophoretic mobility shift assay analysis could not identify any specific transcription factor whose binding could be affected by rs762551. However, a xenobiotic response element (XRE) containing an invariant CACGC core sequence, recognized by $\mathrm{AhR}$, is present in CYP1A2 intron 1 further downstream of the rs762551 site [47]. In the current study, a multiplicative association between having at least one CYP1A2 rs762551 C-allele and at least one $A h R$ Arg554Lys A-allele on the risk for early events in AItreated patients was observed. Helmig et al. reported that the $A h R$ A-allele (Lys) confers lower expression of AhR compared to the G-allele (Arg) [48]. Further, there is cross-talk between $\mathrm{AhR}$ and ER $\alpha$. An animal rat model showed that ligand-activated AhR confers antiestrogenic effects partly due to lower ER $\alpha$ levels in ductal epithelial cells [49]. CYP1A2 is mainly expressed in liver cells but has also been detected in the ER+ breast cancer MCF-7 cell-line after induction [30]. In the current study, neither $A h R$ nor CYP1A2 were associated with prognosis among the patients with ER+ tumors who had not been treated with AIs but either received tamoxifen or no endocrine treatment (data not shown). This suggests that the association of AhR Arg554Lys and CYP1A2 rs762551 on prognosis may be exclusive for the AI-treated patients where the ER is still open as opposed to tamoxifen-treated patients where the ER is blocked. The cross-talk between AhR and ER signaling may be one mechanism behind these findings. Taken together, this 
suggests that $A h R$ G/G carries may have both lower ER $\alpha$ levels and more effective CYP1A2 transcription and expression. This may be especially pronounced in patients with the highly inducible CYP1A2 A/A genotype, since AhR regulates $C Y P 1 A 2$ expression, thus leading to a lower risk for early events during AI-treatment.

In addition to AhR, other cis- or trans acting loci may also regulate CYP1A2 gene expression levels [50]. The CYP1A2 enzyme activity level and gene expression is clustered with CYP2C8, CYP2C9, and CYP3A4 [51]. Further, CYP1A2 share a common promoter with $C Y P 1 A 1$ [29]. However, CYP1A1, CYP2C8, CYP2C9, and CYP3A4 were not significant in the analysis based on the DMET ${ }^{\mathrm{Tx}}$ chip data, therefore no further analyses was performed here.

While the role of CYP1A2 rs762551 with respect to breast cancer risk seems weak or non-significant [52], unless coffee consumption was taken into account $[34,53]$. A meta-analysis showed that the association between the AhR Arg554Lys and breast cancer risk differs between studies of women with different ethnicities, although the overall result was no association [54]. The CYP1A2 rs762551 is associated with the metabolism of several drugs and also with efficacy and toxicity [43]. Although the mechanism behind the finding of the present study is not fully understood, the current study provides new insight into how the CYP1A2 rs762551 combined with the functional $A h R$ Arg554Lys variant is linked to prognosis in AI-treated breast cancer patients. Moreover, AhR appears to be involved in the regulation of CYP19A1 both in ovarian and adrenocortical cells via different mechanisms [55]. Further mechanistic and translational studies of the AhR and CYP1A1/2 signaling pathway with respect to CYP19A1 regulation and AI-response are therefore warranted.

In the present study, a special focus was placed on CYP19A1 since aromatase is the target of AIs. However, genotypes, haplotypes, and diplotypes of CYP19A1 SNPs rs4646, rs10046, Aro1, and Aro2 were not significantly associated with a risk of early breast cancer events in the AI-treated patients. These results are in line with the recent study by of Leyland-Jones et al. [11] that investigated CYP19A1 tumor genotype data in relation to endocrine treatment response in the BIG 1-98 trial, but in contrast to a previous study on genomic CYP19A1 genotypes regarding the risk of recurrence in AI-treated patients [13]. In the recent abstract by Umamasheran et al., an increased risk for breast cancer recurrence was observed among 191 Indian letrozole-treated $\mathrm{T} / \mathrm{T}$ carriers of rs4646 [13]. As mentioned in the background, several SNPs in CYP19A1 have previously been associated with AI response in neoadjuvant and metastatic settings. The rs4646 is among the most frequently studied SNPs. The A-allele has previously been associated with longer time to progression in the metastatic setting [20] but with poor response in the neoadjuvant setting [22]. The differences may be due to ethnicity, different study types, and small study populations. Furthermore, different types of AIs may yield different results. The majority of the patients included in the present study had received anastrozole. A recent study by Lunardi et al. reported no association between plasma estrone concentrations during treatment with letrozole and rs4646 or rs10046 [56]. Since circulating estrone levels may influence the risk for early events in breast cancer, this is in line with the findings of no significant association between these SNPs and early events in the present study.

In the present study, there was a significant interaction between CYP1A2 rs762551 and CYP19A1 rs4646 with a high risk for breast cancer events, especially within five years, among patients with any C-allele of CYP1A2 rs762551 and $\mathrm{C} / \mathrm{C}$-carriers of CYP19A1 rs4646. This subgroup was quite small, and the results need to be interpreted with caution. There was no linkage between CYP19A1 rs4646 and AhR Arg554Lys that could explain the results (data not shown). All findings in the present study warrant validation, preferably within a randomized clinical trial. In such a trial, it would be possible to elucidate whether these genotypes are associated with AI-response. If so, this could guide selection of endocrine treatment for more personalized medicine in the clinical setting.

The Skåne University Hospital in Lund has a catchment area that includes almost 300,000 inhabitants. This study is population-based since patients with breast cancer diagnoses are not referred to other hospitals for surgery. The vast majority of the patients who are diagnosed in Lund are Swedes, but no data on ethnicity was collected. Thus, studies with different study populations are warranted. Since the subset of the cohort that was analyzed with the DMET ${ }^{\mathrm{m}}$ chip also was part of the extended cohort from which they originated, all findings warrant validation in an independent cohort. Further, the follow-up period was relatively short-patients with ER+ tumors tend to relapse later [57]. Therefore, the long-term effects of CYP1A2 rs762551 or CYP19A1 rs4646 could not be evaluated. However, the main impact of these SNPs was observed during the 5-year period when endocrine treatment is administered. This suggests that these SNPs may be involved in primary rather than acquired resistance.

\section{Conclusions}

This study identified a new potential AI-treatment predictive marker in CYP1A2 rs762551 for early breast cancer events, and the results indicate that 
CYP1A2 rs762551 in combination with CYP19A1 rs4646 or AhR Arg554Lys may yield even better treatment prediction. The results of the current study indicate that CYP1A2 rs762551 and the $A h R$ signaling pathway merit further study for AI-response. If confirmed, these results may provide a way to more personalized medicine.

\begin{abstract}
Abbreviations
ADME-related genes: absorption, distribution, metabolism, and elimination-related genes; AhR: aryl hydrocarbon receptor; Al: aromatase inhibitors; BMl: body mass index; CYP: cytochrome P450; DMET: drug metabolism enzymes and transporters; DNA: deoxyribonucleic acid; ER: estrogen receptor; IQR: interquartile range; MAF: minor allele frequency; PgR: progesterone receptor; SNP: single nucleotide polymorphism; WHR: waist-to-hip ratio; XRE: xenobiotic response element.
\end{abstract}

\section{Competing interests}

The authors declare that they have no competing interests.

\section{Authors' contributions}

MS has been involved in acquisition of data, analysis and interpretation of data, in drafting the manuscript, and has revised the manuscript critically for important intellectual content. SV performed statistical analysis of DMET ${ }^{\mathrm{m}}$ chip data and has revised the manuscript critically for important intellectual content. AM has been involved in acquisition of data, analysis and interpretation of data, and in revising the manuscript critically for important intellectual content. $\mathrm{Cl}$ and $\mathrm{CR}$ have been involved in conception and design of the study, analysis and interpretation of data, and have revised the manuscript critically for important intellectual content. $\mathrm{HJ}$ has been involved in acquisition of data, in conception and design of the study, analysis and interpretation of data, in drafting the manuscript, and has revised the manuscript critically for important intellectual content. All authors have read and approved the final version of the manuscript.

\section{Acknowledgements}

We thank our research nurses Maj-Britt Hedenblad, Helén Thell, Karin Henriksson, Annette Möller, Jessica Åkesson, and Linda Ågren. We thank Nils-Gunnar Lundin and Kristina Lövgren for processing the blood samples, Sol-Britt Olsson and Maria Hjertberg for blood processing and DNA extraction, and Erika Bågeman for blood processing, DNA extraction, and sequencing of CYP1A2*1F. We thank Maria Hjertberg and Maria Henningson for data entry. We also thank SCIBLU genomics, Jeanette Valcich for DNA concentration measurements, and Pia Chassale and Ingrid Magnusson Rading for the DMET ${ }^{T M}$ analyses.

\section{Grant Support}

This study was supported by grants from The Swedish Cancer Society, The Swedish Research Council, The Medical Faculty at Lund University, Mrs. Berta Kamprad's Foundation, The Gunnar Nilsson Foundation, VINNOVA, The South Swedish Health Care Region (Region Skåne ALF), Lund Hospital Fund, The Swedish Breast Cancer Group, Gustav V Jubileumsfond, and Märta Winkler's Fund.

\section{Author details}

Department of Clinical Sciences, Lund, Division of Oncology and Pathology, Lund University, Lund, Sweden. ${ }^{2}$ CREATE Health and Department of Immunotechnology, Lund University, Medicon Village, Lund, Sweden. ${ }^{3}$ Department of Clinical Sciences, Lund, Division of Surgery, Lund University and Skåne University Hospital, Lund, Sweden.

\section{Received: 8 October 2015 Accepted: 17 March 2016}

\section{Published online: 31 March 2016}

\section{References}

1. Jemal A, Bray F, Center MM, Ferlay J, Ward E, Forman D. Global cancer statistics. CA Cancer J Clin. 2011;61(2):69-90.

2. Fernö M, Borg A, Johansson U, Norgren A, Olsson H, Ryden S, Sellberg G. Estrogen and progesterone receptor analyses in more than 4,000 human breast cancer samples. A study with special reference to age at diagnosis and stability of analyses. Southern Swedish Breast Cancer Study Group. Acta Oncol. 1990;29(2):129-35.

3. Hou N, Huo D. A trend analysis of breast cancer incidence rates in the United States from 2000 to 2009 shows a recent increase. Breast Cancer Res Treat. 2013;138(2):633-41.

4. Miller WR, Larionov AA. Understanding the mechanisms of aromatase inhibitor resistance. Breast Cancer Res. 2012;14(1):201.

5. Jernström H, Bågeman E, Rose C, Jönsson PE, Ingvar C. CYP2C8 and CYP2C9 polymorphisms in relation to tumour characteristics and early breast cancer related events among 652 breast cancer patients. Br J Cancer. 2009:101(11):1817-23.

6. Markkula A, Hjertberg M, Rose C, Ingvar C, Jernstrom H. No association found between CYP2D6 genotype and early breast cancer events in tamoxifen-treated patients. Acta Oncol. 2014;53(2):195-200.

7. Jung JA, Lim HS. Association between CYP2D6 genotypes and the clinical outcomes of adjuvant tamoxifen for breast cancer: a meta-analysis. Pharmacogenomics. 2014:15(1):49-60.

8. Lum DW, Perel P, Hingorani AD, Holmes MV. CYP2D6 genotype and tamoxifen response for breast cancer: a systematic review and meta-analysis. PLoS One. 2013;8(10):e76648.

9. Cronin-Fenton DP. Damkier P. Lash TL. Metabolism and transport of tamoxifen in relation to its effectiveness: new perspectives on an ongoing controversy. Future Oncol. 2014;10(1):107-22.

10. Blackburn $\mathrm{HL}$, Ellsworth $\mathrm{DL}$, Shriver CD, Ellsworth RE. Role of cytochrome P450 genes in breast cancer etiology and treatment: effects on estrogen biosynthesis, metabolism, and response to endocrine therapy. Cancer Causes Control. 2015;26(3):319-32.

11. Leyland-Jones B, Gray KP, Abramovitz M, Bouzyk M, Young B, Long B, Kammler R, Dell'Orto P, Biasi MO, Thurlimann B, et al. CYP19A1 polymorphisms and clinical outcomes in postmenopausal women with hormone receptor-positive breast cancer in the BIG 1-98 trial. Breast Cancer Res Treat. 2015:151(2):373-84.

12. Miller WR. Aromatase inhibitors and breast cancer. Minerva Endocrinol. 2006:31(1):27-46.

13. Umamaheswaran GK D, Dubashi B, Sathyanarayana Reddy K, Adithan CAD S. Polymorphisms in CYP19A1 gene predicts relapse and survival in postmenopausal breast cancer patients treated with adjuvant letrozole. In: 9th International Conference of Anticancer Research. Sithonia: International Institute of Anticancer Research (J.G. Delinasios); 2014.

14. Kaufmann M, von Minckwitz G, Mamounas EP, Cameron D, Carey LA, Cristofanilli M, Denkert C, Eiermann W, Gnant M, Harris JR, et al. Recommendations from an international consensus conference on the current status and future of neoadjuvant systemic therapy in primary breast cancer. Ann Surg Oncol. 2012;19(5):1508-16.

15. Smith IE, Dowsett M, Ebbs SR, Dixon JM, Skene A, Blohmer JU, Ashley SE, Francis S, Boeddinghaus I, Walsh G. Neoadjuvant treatment of postmenopausal breast cancer with anastrozole, tamoxifen, or both in combination: the Immediate Preoperative Anastrozole, Tamoxifen, or Combined with Tamoxifen (IMPACT) multicenter double-blind randomized trial. J Clin Oncol. 2005;23(22):5108-16.

16. Mouridsen $\mathrm{H}$, Gershanovich M, Sun Y, Perez-Carrion R, Boni C, Monnier A Apffelstaedt J, Smith R, Sleeboom HP, Jaenicke F, et al. Phase III study of letrozole versus tamoxifen as first-line therapy of advanced breast cancer in postmenopausal women: analysis of survival and update of efficacy from the International Letrozole Breast Cancer Group. J Clin Oncol. 2003;21(11):2101-9.

17. Osborne CK, Schiff R. Mechanisms of endocrine resistance in breast cancer. Annu Rev Med. 2011:62:233-47.

18. Long JR, Kataoka N, Shu XO, Wen W, Gao YT, Cai Q, Zheng W. Genetic polymorphisms of the CYP19A1 gene and breast cancer survival. Cancer Epidemiol Biomarkers Prev. 2006;15(11):2115-22.

19. Fasching PA, Loehberg CR, Strissel PL, Lux MP, Bani MR, Schrauder M, Geiler S, Ringleff K, Oeser S, Weihbrecht S, et al. Single nucleotide polymorphisms of the aromatase gene (CYP19A1),

HER2/neu status, and prognosis in breast cancer patients. Breast Cancer Res Treat. 2008:112(1):89-98.

20. Artigalas O, Vanni T, Hutz MH, Ashton-Prolla P, Schwartz IV. Influence of CYP19A1 polymorphisms on the treatment of breast cancer with aromatase inhibitors: a systematic review and meta-analysis. BMC Med. 2015;13(1):139.

21. Wang L, Ellsworth KA, Moon I, Pelleymounter LL, Eckloff BW, Martin YN, Fridley BL, Jenkins GD, Batzler A, Suman VJ, et al. Functional genetic 
polymorphisms in the aromatase gene CYP19 vary the response of breast cancer patients to neoadjuvant therapy with aromatase inhibitors. Cancer Res. 2010;70(1):319-28.

22. Garcia-Casado Z, Guerrero-Zotano A, Llombart-Cussac A, Calatrava A, Fernandez-Serra A, Ruiz-Simon A, Gavila J, Climent MA, Almenar S, Cervera-Deval J, et al. A polymorphism at the 3'-UTR region of the aromatase gene defines a subgroup of postmenopausal breast cancer patients with poor response to neoadjuvant letrozole. BMC Cancer. 2010;10:36.

23. Tsuchiya Y, Nakajima M, Yokoi T. Cytochrome P450-mediated metabolism of estrogens and its regulation in human. Cancer Lett. 2005;227(2):115-24.

24. Kamdem LK, Liu Y, Stearns V, Kadlubar SA, Ramirez J, Jeter S, Shahverdi K, Ward BA, Ogburn E, Ratain MJ, et al. In vitro and in vivo oxidative metabolism and glucuronidation of anastrozole. Br J Clin Pharmacol. 2010;70(6):854-69.

25. Buzdar AU, Robertson JF, Eiermann W, Nabholtz JM. An overview of the pharmacology and pharmacokinetics of the newer generation aromatase inhibitors anastrozole, letrozole, and exemestane. Cancer. 2002;95(9):2006-16.

26. Jeong S, Woo MM, Flockhart DA, Desta Z. Inhibition of drug metabolizing cytochrome P450s by the aromatase inhibitor drug letrozole and its major oxidative metabolite 4,4'-methanol-bisbenzonitrile in vitro. Cancer Chemother Pharmacol. 2009;64(5):867-75.

27. Grimm SW, Dyroff MC. Inhibition of human drug metabolizing cytochromes P450 by anastrozole, a potent and selective inhibitor of aromatase. Drug Metab Dispos. 1997;25(5):598-602.

28. Kamdem LK, Flockhart DA, Desta Z. In vitro cytochrome P450-mediated metabolism of exemestane. Drug Metab Dispos. 2011;39(1):98-105.

29. Jiang Z, Dalton TP, Jin L, Wang B, Tsuneoka Y, Shertzer HG, Deka R, Nebert DW. Toward the evaluation of function in genetic variability: characterizing human SNP frequencies and establishing BAC-transgenic mice carrying the human CYP1A1_CYP1A2 locus. Hum Mutat. 2005;25(2):196-206.

30. Li W, Harper PA, Tang BK, Okey AB. Regulation of cytochrome P450 enzymes by aryl hydrocarbon receptor in human cells: CYP1A2 expression in the LS180 colon carcinoma cell line after treatment with 2,3,7,8-tetrachlorodibenzo-pdioxin or 3-methylcholanthrene. Biochem Pharmacol. 1998;56(5):599-612.

31. Burmester JK, Sedova M, Shapero MH, Mansfield E. DMET microarray technology for pharmacogenomics-based personalized medicine. Methods Mol Biol. 2010;632:99-124.

32. Simonsson M, Söderlind V, Henningson M, Hjertberg M, Rose C, Ingvar C, Jernström $\mathrm{H}$. Coffee prevents early events in tamoxifen-treated breast cancer patients and modulates hormone receptor status. Cancer Causes Control. 2013:24(5):929-40

33. Lundin KB, Henningson M, Hietala M, Ingvar C, Rose $C$, Jernström $H$. Androgen receptor genotypes predict response to endocrine treatment in breast cancer patients. Br J Cancer. 2011;105(11):1676-83.

34. Bågeman E, Ingvar C, Rose C, Jernström H. Coffee consumption and CYP1A2*1 F genotype modify age at breast cancer diagnosis and estrogen receptor status. Cancer Epidemiol Biomarkers Prev. 2008;17(4):895-901.

35. Simonsson M, Markkula A, Bendahl PO, Rose C, Ingvar C, Jernström H. Pre- and postoperative alcohol consumption in breast cancer patients: impact on early events. Springerplus. 2014;3:261.

36. Database of Single Nucleotide Polymophisms (dbSNP). In., vol. dbSNP: rs4646, rs10046, rs4775936, rs10459592. Build ID: 52/137;111/137;119/137. National Center for Biotechnology information, National Library of Medicine: Bethesda; 2012. http://www.ncbi.nlm.nih.gov/SNP/

37. Benjamini $Y$, Hochberg $Y$. Controlling the false discovery rate: a practical and powerful approach to multiple testing. J R Stat Soc Ser B (Methodological). 1995;57(1):289-300

38. Dupont WD, Plummer Jr WD. Power and sample size calculations for studies involving linear regression. Control Clin Trials. 1998;19(6):589-601.

39. McShane LM, Altman DG, Sauerbrei W, Taube SE, Gion M, Clark GM. REporting recommendations for tumour MARKer prognostic studies (REMARK). Eur J Cancer. 2005;41(12):1690-6.

40. Sim SC, Kacevska M, Ingelman-Sundberg M. Pharmacogenomics of drug-metabolizing enzymes: a recent update on clinical implications and endogenous effects. Pharmacogenomics J. 2013;13(1):1-11.

41. Schneider J, Huh MM, Bradlow HL, Fishman J. Antiestrogen action of 2-hydroxyestrone on MCF-7 human breast cancer cells. J Biol Chem. 1984;259(8):4840-5.

42. Klug TL, Bågeman E, Ingvar C, Rose C, Jernström H. Moderate coffee and alcohol consumption improves the estrogen metabolite profile in adjuvant treated breast cancer patients: a pilot study comparing pre- and postoperative levels. Mol Genet Metab. 2006;89(4):381-9.

43. Zhou SF, Yang LP, Zhou ZW, Liu YH, Chan E. Insights into the substrate specificity, inhibitors, regulation, and polymorphisms and the clinical impact of human cytochrome P450 1A2. AAPS J. 2009;11(3):481-94.

44. Sachse C, Brockmoller J, Bauer S, Roots I. Functional significance of a C->A polymorphism in intron 1 of the cytochrome P450 CYP1A2 gene tested with caffeine. Br J Clin Pharmacol. 1999;47(4):445-9.

45. Djordjevic N, Ghotbi R, Jankovic S, Aklillu E. Induction of CYP1A2 by heavy coffee consumption is associated with the CYP1A2 -163C > A polymorphism. Eur J Clin Pharmacol. 2010;66(7):697-703.

46. Ingelman-Sundberg M, Sim SC, Gomez A, Rodriguez-Antona C. Influence of cytochrome P450 polymorphisms on drug therapies: pharmacogenetic, pharmacoepigenetic and clinical aspects. Pharmacol Ther. 2007;116(3):496-526.

47. Aklillu E, Carrillo JA, Makonnen E, Hellman K, Pitarque M, Bertilsson L, Ingelman-Sundberg M. Genetic polymorphism of CYP1A2 in Ethiopians affecting induction and expression: characterization of novel haplotypes with single-nucleotide polymorphisms in intron 1. Mol Pharmacol. 2003; 64(3):659-69.

48. Helmig S, Seelinger JU, Dohrel J, Schneider J. RNA expressions of AHR, ARNT and CYP1B1 are influenced by AHR Arg554Lys polymorphism. Mol Genet Metab. 2011;104(1-2):180-4.

49. Helle J, Bader MI, Keiler AM, Zierau O, Vollmer G, Chittur SV, Tenniswood M, Kretzschmar G. Cross-talk in the female rat mammary gland: influence of aryl hydrocarbon receptor on estrogen receptor signaling. Environ Health Perspect. 2015. [Epub ahead of print]

50. Jiang Z, Dragin N, Jorge-Nebert LF, Martin MV, Guengerich FP, Aklillu E, Ingelman-Sundberg M, Hammons GJ, Lyn-Cook BD, Kadlubar FF, et al. Search for an association between the human CYP1A2 genotype and CYP1A2 metabolic phenotype. Pharmacogenet Genomics. 2006;16(5):359-67.

51. Yang X, Zhang B, Molony C, Chudin E, Hao K, Zhu J, Gaedigk A, Suver C, Zhong $\mathrm{H}$, Leeder JS, et al. Systematic genetic and genomic analysis of cytochrome P450 enzyme activities in human liver. Genome Res. 2010;20(8): 1020-36.

52. Wang H, Zhang Z, Han S, Lu Y, Feng F, Yuan J. CYP1A2 rs762551 polymorphism contributes to cancer susceptibility: a meta-analysis from 19 case-control studies. BMC Cancer. 2012;12:528.

53. Kotsopoulos J, Ghadirian P, El-Sohemy A, Lynch HT, Snyder C, Daly M, Domchek S, Randall S, Karlan B, Zhang P, et al. The CYP1A2 genotype modifies the association between coffee consumption and breast cancer risk among BRCA1 mutation carriers. Cancer Epidemiol Biomarkers Prev. 2007;16(5):912-6.

54. Li Y, Qin HZ, Song Q, Wu XD, Zhu JH. Lack of association between the aryl hydrocarbon receptor rs2066853 polymorphism and breast cancer: A meta-analysis on Ahr polymorphism and breast cancer. Genet Mol Res. 2015;14(4):16162-8.

55. Li LA. Polychlorinated biphenyl exposure and CYP19 gene regulation in testicular and adrenocortical cell lines. Toxicol In Vitro. 2007;21(6):1087-94.

56. Lunardi G, Piccioli P, Bruzzi P, Notaro R, Lastraioli S, Serra M, Marroni P, Bighin C, Mansutti M, Puglisi F, et al. Plasma estrone sulfate concentrations and genetic variation at the CYP19A1 locus in postmenopausal women with early breast cancer treated with letrozole. Breast Cancer Res Treat. 2013;137(1):167-74.

57. Osborne CK, Yochmowitz MG, Knight 3rd WA, McGuire WL. The value of estrogen and progesterone receptors in the treatment of breast cancer. Cancer. 1980;46(12 Suppl):2884-8.

\section{Submit your next manuscript to BioMed Central and we will help you at every step:}

- We accept pre-submission inquiries

- Our selector tool helps you to find the most relevant journal

- We provide round the clock customer support

- Convenient online submission

- Thorough peer review

- Inclusion in PubMed and all major indexing services

- Maximum visibility for your research

Submit your manuscript at www.biomedcentral.com/submit
Biomed Central 